Loisir et Société / Society and Leisure

\title{
L'accompagnement-citoyen en soutien à la participation sociale des aînés ayant un traumatisme craniocérébral
}

Marie-Josée Levert, Hélène Lefebvre, Mélanie Levasseur \& Isabelle Gélinas

To cite this article: Marie-Josée Levert, Hélène Lefebvre, Mélanie Levasseur \& Isabelle Gélinas (2019) L'accompagnement-citoyen en soutien à la participation sociale des aînés ayant un traumatisme craniocérébral, Loisir et Société / Society and Leisure, 42:1, 91-107

To link to this article: https://doi.org/10.1080/07053436.2019.1582915

曲 Published online: 09 May 2019.

Submit your article to this journal $\complement$

View Crossmark data $\longleftarrow$ 


\title{
L'accompagnement-citoyen en soutien à la participation sociale des aînés ayant un traumatisme craniocérébral
}

\author{
Marie-Josée Levert ${ }^{\mathrm{a}, \mathrm{b} *}$, Hélène Lefebvre ${ }^{\mathrm{a}, \mathrm{b}}$, Mélanie Levasseur ${ }^{\mathrm{c}}$ et Isabelle Gélinas ${ }^{\mathrm{b}, \mathrm{d}}$ \\ ${ }^{a}$ Faculté des sciences infirmières, Université de Montréal, Montréal, Québec, Canada; ${ }^{b}$ Centre de \\ recherche interdisciplinaire en réadaptation du Montréal métropolitain (CRIR), Montréal, Québec, \\ Canada; ${ }^{\circ}$ École de réadaptation, Faculté de médecine et des sciences de la santé, Université de \\ Sherbrooke, Sherbrooke, QC, Canada; ${ }^{d}$ School of Physical and Occupational Therapy, McGill \\ University, Montréal, Québec, Canada
}

(Received 16 July 2018; accepted 4 January 2019)

\begin{abstract}
Highly valued by seniors, community integration has beneficial effects on their biopsychosocial health. However, not all of them have the same opportunities to engage in social participation activities, particularly those with traumatic brain injury (TBI) who are more likely to be limited in their abilities to participate in the life of the community. The most common initiatives to help these people consist of human assistance to overcome their limitations and which do not focus on skills promotion. In order to optimize the available services and resources, personalized citizen assistance for community integration (APIC) has been developed, implemented, and evaluated. This article documents the impact of APIC on the community integration of seniors with TBI. A thematic content analysis was conducted on data from semi-structured interviews with participants and from attendant logbooks. The results show three major spin-offs from APIC: better insurance and a greater sense of autonomy, a renewed taste for investing in meaningful leisure activities, and greater openness to new relationship opportunities.
\end{abstract}

Keywords: personalized citizen assistance; seniors; community integration

Hautement valorisée par les aînés, la participation sociale a des effets bénéfiques sur leur santé biopsychosociale. Tous n'ont pas cependant les mêmes opportunités pour s'investir dans ce type d'activités; particulièrement ceux ayant un traumatisme craniocérébral (TCC) qui sont plus susceptibles d'être limités dans leurs capacités à participer à la vie de la communauté. Les initiatives les plus courantes pour aider ces personnes consistent en une assistance humaine visant à pallier leurs limitations et ne misent pas, en priorité, sur la promotion de leurs compétences. Afin d'optimiser l'aide reçue, un accompagnementcitoyen personnalisé à l'intégration communautaire (APIC) a été développé, implanté et évalué. Cet article documente l'impact de l'APIC sur la participation sociale d'aînés ayant un TCC. Une analyse de contenu thématique a été réalisée sur les données issues d'entrevues semi-dirigées auprès des participants et des journaux de bord des accompagnateurs. Les résultats montrent trois grandes retombées de l'APIC : une meilleure assurance et un plus grand sentiment d'autonomie, un goût retrouvé de s'investir dans des activités de loisirs signifiantes, et une plus grande ouverture vers de nouvelles possibilités de relations.

Mots clés : accompagnement-citoyen; aînés; participation sociale

\section{Introduction}

Pour pouvoir se développer de manière optimale, toute personne doit être reconnue par les membres de sa collectivité (Pourtois et Desmet, 2014). Ce processus repose

*Corresponding author. Email: mj.levert@umontreal.ca 
notamment sur la possibilité d'exercer des rôles sociaux (vie communautaire, travail, éducation) et de réaliser des activités offrant des occasions de s'épanouir dans sa vie quotidienne, ses loisirs et ses interactions sociales (Hammel et al., 2008; Hardaker, 2011; Levasseur, Richard, Gauvin, et Raymond, 2010; McColl et al., 1998; Pourtois et Desmet, 2014; Raymond, Gagné, Sévigny, et Tourigny, 2008; Therriault, Lefebvre, Guindon, Levert, et Briand, 2013). Hautement valorisée par les aînés, la participation sociale est reconnue pour avoir des effets bénéfiques de protection de l'état de santé physiologique, du fonctionnement cognitif, du maintien des habiletés sociales et de la qualité de vie (Buffel, Phillipson, et Scharf, 2012; Dahan-Oliel, Gélinas, et Mazer, 2008; Organisation mondiale de la santé [OMS], 2007; Raymond et al., 2008).

Tous n'ont pas cependant les mêmes opportunités pour s'investir dans des activités de participation sociale en fonction de leurs caractéristiques et l'environnement dans lequel ils évoluent. À cet égard, les aînés ayant subi un traumatisme craniocérébral (TCC) représentent un groupe de la population âgée à risque d'exclusion sociale, car plus susceptible d'être limités dans leurs capacités à participer activement à la vie de la communauté. En effet, au cours des 10 dernières années, la prévalence du TCC a en effet augmenté de façon remarquable dans ce groupe de la population (Faul, $\mathrm{Xu}$, Wald, et Coronado, 2010; Leblanc, de Guise, Gosselin, et Feyz, 2006), les personnes de plus de 75 ans représentent la majorité des cas de consultation à l'urgence, d'hospitalisation et de décès associés à un TCC (Faul et al., 2010), le plus souvent consécutifs à une chute (62\% des cas selon le Center for Disease Control, 2006). Comparativement aux adultes plus jeunes, les aînés sont aussi plus susceptibles de voir leur autonomie fonctionnelle se détériorer, d'abord du fait des séquelles physiques, cognitives, affectives et comportementales du TCC (Société d'assurance-automobile du Québec [SAAQ], 2003; Winkler, Unsworth, et Sloan, 2006), puis en raison du déclin accéléré découlant du TCC (Cisneros, 2012; Frankel, Marwitz, Cifu, Kreutzer, Englander, et Rosenthal, 2006; McIntyre, Janzen, et Teasell, 2014; McIntyre, Mehta, Janzen, Aubut, et Teasell, 2013; Sendroy-Terrill, Whiteneck, et Brooks, 2010; Yi, 2013). Ces séquelles placent les ainés à risque de subir de nouvelles chutes (Benker, 2003) et limitent considérablement leur capacité à reprendre leurs activités habituelles, à s'engager de nouveau dans des occupations et dans des relations interpersonnelles signifiantes (Hardaker, 2011; Yi et Dams-O'Connor, 2013).

Certaines ressources de soutien sont actuellement offertes aux personnes ayant subi un TCC, sans égard à l'âge (Lamontagne, Ouellet, et Simard, 2009; McCluskey, 2000). Peu développées malgré les besoins criants, elles peuvent prendre la forme d'un parrainage, de visites d'amitié, d'une aide à domicile ou à la réalisation de loisirs, et s'actualisent dans différents domaines de vie : éducation, travail, vie communautaire, activités de la vie quotidienne, loisirs (Lamontagne et al., 2009; McCluskey, 2000; Raymond et al., 2008; Table de concertation en accompagnement de Montréal, 2012). Or, la plupart se préoccupent généralement de pallier ou de suppléer aux limitations de la personne (Table de concertation en accompagnement de Montréal, 2012) et ne visent pas prioritairement la promotion de ses forces, le développement de ses compétences ou son autodétermination, ni ne sont systématiquement centrés sur ses valeurs, ses intérêts et son projet de vie. Il est pourtant admis que, pour véritablement agir sur la participation sociale de populations fragilisées, il est essentiel d'optimiser leurs capacités et celles de leur environnement (Hammel et al., 2008; Levasseur et al., 2010; McColl et al., 1998; Therriault et al., 2013) et de les inclure comme des partenaires-experts de leur participation sociale en 
considérant leurs besoins, leurs objectifs et la manière dont elles souhaitent s'investir dans l'actualisation de leur projet de vie (Lefebvre et al., 2013; Levert et al., 2017). Afin d'optimiser l'aide reçue, un accompagnement-citoyen personnalisé à l'intégration communautaire (APIC) a été expérimenté et évalué auprès d'aînés ayant un TCC. Cet article vise à documenter l'impact longitudinal de l'APIC sur leur participation sociale.

\section{L'accompagnement-citoyen personnalisé à l'intégration communautaire (APIC)}

En complémentarité et en continuité avec les ressources existantes, l'APIC offre un soutien aux personnes vivant des difficultés sur le plan de leur participation sociale par l'entremise d'un mentorat à la réalisation des activités de la vie quotidienne et de loisirs valorisées par elles (Lefebvre et al., 2010, Lefebvre et al., 2013; Lefebvre et Levert, 2010; Lefebvre, Levert et Khelia, 2011; Levasseur et al., 2016; Levert et Lefebvre, 2014; Levert et al., 2017; Therriault et al., 2013). La prémisse à la base de l'approche est qu'en participant à des activités signifiantes, la personne acquière de nouveaux savoirs et savoir-faire, développe ses compétences et sa confiance en ses ressources, ce qui lui permet de s'autodéterminer dans son projet de participation sociale. Pour ce faire, l'APIC engage un citoyen de la communauté (qui n'est ni un proche, ni un intervenant, ni un pair-aidant), mais bien un citoyen ayant l'intérêt de s'investir dans le projet de participation sociale d'un autre citoyen. Chaque candidat accompagnateur est soumis à un entretien d'embauche et doit manifester des habiletés sur le plan du savoir-être (intérêt vis-à-vis de l'accompagnement, du soutien, écoute, empathie, respect) indispensable à l'accompagnement. Formé, rémunéré et encadré, l'accompagnateur-citoyen a pour mandat d'amener la personne à jeter un regard réflexif sur sa participation sociale, à se fixer des buts et à réaliser les activités de son choix qu'elle valorise, en lui offrant des séances de stimulations personnalisées dans le milieu de vie à raison de trois heures/semaine. Le but visé est d'amener la personne à développer, avec l'aide de son accompagnateur-citoyen, sa capacité à mobiliser ses forces et ses ressources personnelles et celles de son environnement. Plusieurs études ont démontré les effets positifs de l'APIC sur la réalisation d'activités sociales et de loisirs, sur la satisfaction quant à la participation sociale et le mieux-être d'adultes ayant un TCC (Lefebvre et al., 2013) ou un TCC léger (Perroux, Lefebvre, Levert, et Malo, 2013), d'aînés en perte d'autonomie fonctionnelle (Levasseur et al., 2016), et d'adultes ayant des limitations motrices, intellectuelles, cognitives, sensorielles, affectives ou communicationnelles (Levert, Gélinas, et Lefebvre, 2016).

\section{Cadre de référence}

L'article s'appuie sur le concept de participation sociale tel qu'opérationnalisé par Hardaker (2011) dans trois grandes sphères d'activités.

(1) La vie autonome et indépendante au quotidien : pour pouvoir participer socialement, l'aîné doit pouvoir évoluer dans un contexte de vie facilitant qui l'amènera à relever ses propres défis. Il doit être en mesure d'accomplir ses activités de la vie quotidienne (AVQ) de la façon la plus autonome et indépendante possible (Raymond et al., 2008). 
(2) Les occupations et loisirs : l'aîné doit pouvoir s'engager dans des activités récréatives, sociales, culturelles et spirituelles valorisées par lui, cet engagement pouvant se traduire de différentes façons selon ses capacités et ses intérêts (OMS, 2007).

(3) Les relations avec les autres : le maintien et la consolidation des relations familiales et sociales significatives a pour effet de motiver l'aîné à s'investir dans sa collectivité; la présence et le soutien d'autrui étant par ailleurs considérés comme des leviers fondamentaux de la participation sociale (OMS, 2007; Raymond et al., 2008). À la lumière de ce cadre de référence, la participation sociale des aînés est optimale lorsqu'ils accomplissent les activités de leur choix, de manière autonome et indépendante, dans un contexte de relations humaines positives et mobilisatrices et dans un environnement personnel et social vitalisant et accueillant (Therriault et al., 2013).

\section{Méthodologie}

Une étude longitudinale a été réalisée afin d'évaluer l'impact de l'APIC sur la participation sociale des aînés ayant subi un TCC. Avec le soutien d'un groupe d'experts, l'APIC a été adapté au contexte des aînés ayant un TCC, puis expérimenté et évalué sur une période de 12 mois. Pour répondre à l'objectif de l'étude, un devis d'études de cas multiple (Stake, 2005) a été mis en place. Chaque cas est défini comme un aîné ayant un TCC, son histoire, son environnement (physique, familial et social) et son évolution à travers l'intervention APIC en fonction des séquelles spécifiques qu'il associe à son TCC. L'étude s'inscrit dans le paradigme constructiviste (Mucchielli, 2005) offrant des occasions d'apprendre, de réfléchir et de considérer le point de vue de l'aîné ayant un TCC sur le soutien d'accompagnement reçu afin de dégager les meilleures façons de faire pour favoriser sa participation sociale. L'étude a reçu l'approbation éthique des établissements partenaires et les normes édictées par l'Énoncé de politiques des Trois Conseils en recherche du Canada ont été respectées.

\section{Échantillon}

Les participants ont été recrutés de façon intentionnelle à partir de critères précis et dans un souci de diversification (McLean, Jarus, Hubley, et Jongbloed, 2014), lesquels incluaient : avoir subi un TCC léger ou modéré, avoir 65 ans et plus, vivre à domicile ou dans une ressource intermédiaire. Le recrutement a été réalisé par l'entremise des centres de réadaptation en déficience physique partenaires de la région du Grand Montréal à partir de la consultation d'archives médicales et de références des cliniciens des programmes TCC. L'échantillon est formé de 11 participants (Tableau 1), dont sept femmes et quatre hommes, âgés de 65 ans à 96 ans (moyenne $=78$ ans). La forte majorité (7) est issue de communautés culturelles (ex. Chili, Italie, Grèce).

La plupart des participants sont atteints d'un TCC léger complexe (résultat 13 à 15 sur l'échelle de Glasgow, scan cérébral positif), survenu depuis deux à neuf ans au moment de la collecte des données (moyenne $=3,5$ ans). Selon les données disponibles (trois données manquantes), leur TCC est le plus souvent causé par une chute $(N=8)$ et de façon isolée, par un accident de la route $(N=1)$. De plus, les participants sont nombreux à présenter des comorbidités (ex. arthrite, AVC, diabètes, problèmes cardiaques) et leur niveau d'autonomie est variable. Certains participants, moins autonomes en termes de soins personnels, reçoivent des services dispensés par des établissements de 
Tableau 1. Échantillon.

\begin{tabular}{lcc}
\hline Caractéristiques & $N$ & $\%$ \\
\hline Sexe & & \\
Femme & 7 & $64 \%$ \\
Homme & 4 & $36 \%$ \\
État civil & 4 & $36 \%$ \\
Marié & 5 & $46 \%$ \\
Divorcé/séparé & 2 & $18 \%$ \\
Veuf & & \\
Type de TCC & & \\
*1 donnée manquante & 1 & $10 \%$ \\
Léger & 6 & $60 \%$ \\
Léger complexe & 3 & $30 \%$ \\
Modéré & & \\
Réseau familial & 1 & $9 \%$ \\
Conjoint(e) & 7 & $64 \%$ \\
Enfant(s) & 3 & $27 \%$ \\
Aucun & & \\
Domicile & 4 & $36 \%$ \\
Résidence pour aînés & 5 & $46 \%$ \\
Propriétaire & 2 & $18 \%$ \\
Locataire & & \\
Nombre d'années depuis le traumatisme & 3 & $27 \%$ \\
Moins de 2 ans & & $64 \%$ \\
Entre 2 à 4 ans & 7 & $9 \%$ \\
Plus de 4 ans & 1 & \\
\hline
\end{tabular}

santé (adaptation des équipements à domicile, aide professionnelle pour se laver, faire le ménage ou pour aider à la prise de médicaments) ou disposent d'une assistance communautaire (service de livraison de repas).

\section{Déroulement des accompagnements}

Les accompagnements ont été d'une durée moyenne de 12 à 18 mois. Accompagnateurs et accompagnés se sont rencontrés sur une base régulière au domicile du participant ou dans un autre lieu à la convenance de chacun, et en réponse à l'activité ou au projet ciblé par le participant. En moyenne, les participants ont bénéficié de 26 rencontres à raison, approximativement, de trois heures par rencontre. Ils ont généralement reçu deux accompagnements par semaine pour les six premiers mois, suivi d'un seul accompagnement par semaine pendant trois mois, puis d'un accompagnement à toutes les deux semaines pendant les trois derniers mois afin de faciliter la transition vers la fin de l'accompagnement.

L'horaire et les activités des rencontres ont été flexibles et ont varié selon les besoins, les prises de décision et le choix des activités paraissant toujours découler des attentes, des circonstances et des capacités des participants. La décision d'activités a parfois été prise d'un commun accord, le participant et son accompagnateur explorant ensemble le type d'activités à réaliser, ou encore, le choix de l'activité a reposé entre les mains du participant, l'accompagnateur s'associant à ses demandes. L'accompagnateur a aussi soumis des propositions d'activités que le participant acceptait ou déclinait, la décision étant prise, à l'occasion, avec l'aide d'un proche lorsque le participant manifestait des signes de replis sur soi importants, principalement dans les premiers mois d'accompagnement. 
La plupart des accompagnements se sont déroulées en français, sauf exception chez deux participants qui ne s'exprimaient qu'en anglais. On compte, au total, huit annulations de séances d'accompagnement pour différents motifs (incapacités de réaliser l'activité planifiée à cause de la pluie, visite de la famille, maladie, rendez-vous médical). Les accompagnements ont été suspendus durant cinq mois pour une participante qui souhaitait éviter les sorties extérieures durant la période hivernale, puis ont repris au cours du printemps.

\section{Collecte des données}

Les données d'évaluation ont été recueillies à quatre reprises (soit avant le début de l'intervention d'accompagnement, puis à trois et six mois, et à la fin de l'intervention, soit 12 mois) par le biais d'entrevues semi-dirigées réalisées auprès des aînés accompagnés et de journaux de bord hebdomadaires tenus par leurs accompagnateurs. Les entrevues semidirigées (durée moyenne de 60 minutes) ont été réalisées au domicile des participants, parfois en présence d'un proche (deux participants) en raison des difficultés communicationnelles (contraintes de langue, aphasie). Elles ont été enregistrées sur bande audio, puis transcrites verbatim. Les entrevues ont été menées à l'aide d'un guide adapté à la clientèle aînée à partir d'une grille validée et développée en s'appuyant sur deux outils de mesure reconnus, soit le Profil d'activités de la vie quotidienne v. 5 (Dutil, Bottari, Vanier, et Rousseau, 2005) et celui des loisirs v.3.0 (Dutil, Bier, et Gaudreault, 2006). Le Profil des AVQ permet d'évaluer les changements perçus par le participant quant à sa participation sociale et sa prise de responsabilité dans ses activités de la vie quotidienne. Le Profil des loisirs permet d'évaluer son niveau d'engagement quant à ses activités de loisirs. Les journaux de bord ont été complétés par l'accompagnateur après chaque rencontre d'accompagnement, pour documenter la progression dans la relation avec l'accompagné et au niveau des activités de participation sociale réalisées, et acheminés au chercheur.

\section{Analyse et interprétation des données}

Une analyse de contenu thématique (Paillé et Mucchielli, 2016) a été effectuée à partir de catégories prédéterminées et émergentes. Les données ont été organisées par participant en fonction des quatre temps de mesure de l'étude, selon les trois grandes sphères d'activités de la participation sociale du cadre opérationnel, puis ont été synthétisées et réunies dans un tableau récapitulatif (situation sociale et de santé, besoins fondamentaux en matière de participation sociale, limitations initialement identifiées et activités de participation sociale mobilisées grâce au soutien de l'accompagnateur). Au terme de l'analyse des données, une estimation de la progression du participant sur le plan de sa participation sociale a été effectuée à partir de la triangulation des données. Le matériel synthétisé a fourni la base pour des analyses transversales. Celles-ci ont permis de dégager les thèmes récurrents, les divergences et les constantes. Un chercheur et une professionnelle de recherche ont assuré la validité interne des analyses par l'obtention de consensus sur la codification et l'interprétation des données.

\section{Résultats}

Les résultats permettent de mieux comprendre les effets de l'APIC chez les aînés ayant un TCC, en dressant un portrait dynamique de l'évolution de leur participation sociale au cours de l'année d'accompagnement. 


\section{État de la participation sociale des aînés avant la prestation de l'APIC}

$\mathrm{Au}$ moment pré-intervention, tous les participants identifiaient de multiples limitations consécutives à leur TCC qui contraignaient leur participation sociale. Ces limitations étaient présentes, même quelques années après le TCC (après quatre ans, en moyenne), et étaient associées par les participants à des atteintes motrices (ex. pertes d'équilibre), sensorielles (ex. troubles de la vision), affectives (ex. irritabilité), cognitives (ex. pertes de mémoire) ou à des dommages physiques connexes au TCC (ex. amputation d'une jambe) (Tableau 2).

Au début de l'intervention APIC, ces limitations constituaient des barrières importantes à la participation sociale de la majorité des participants, et ce, dans les trois sphères d'activités du cadre de référence (AVQ, loisirs, relations). D'abord, la plupart exprimaient, à des degrés divers, des difficultés dans la réalisation des activités de la vie quotidienne, plusieurs subvenant difficilement à leur alimentation (ex. emplettes et préparation des repas), à l'entretien domestique ou à leurs soins personnels, en raison de séquelles du TCC. De plus, de nombreux participants souffraient d'un manque de motivation pour amorcer leur journée, éprouvant des difficultés à sortir du lit, se souciant peu de leur apparence ou ne trouvant plus l'élan pour réaliser leurs activités quotidiennes. Ensuite, tous se sentaient freinés, sur le plan des loisirs, par les séquelles du TCC, exprimant des difficultés à lire, à faire des exercices physiques (vélo stationnaire à la maison, tennis, entraînement, danse, marche), ou à réaliser des activités de loisirs signifiantes (cinéma, télévision, casse-têtes, couture). Enfin, la plupart des participants se disaient restreints dans leurs relations interpersonnelles et sociales, certains hésitant à s'engager dans des activités sociales parce qu'ils éprouvaient des difficultés à suivre les conversations ou à communiquer, ne se sentant plus à la hauteur ou craignant d'être jugés. L'ensemble de ces difficultés sur le plan de la participation sociale était consolidé par des barrières aux déplacements extérieurs, particulièrement liées à des craintes de chuter, qui décourageaient la majorité à sortir. Plusieurs participants témoignaient ainsi d'un manque d'interactions sociales (ex. rétrécissement du cercle social, deuil, veuvage), et exprimaient des impacts néfastes sur leur bien-être.

\section{Effets de l'APIC sur la participation sociale des â̂nés ayant un TCC}

Dans le cadre de la prestation de l'APIC, tous les participants ont été en mesure de cibler un projet d'accompagnement et ont progressivement réalisé des activités signifiantes.

Tableau 2. Séquelles du TCC identifiées par les participants.

\begin{tabular}{lcc}
\hline Définitions - Séquelles & $N$ & $\%$ \\
\hline Fatigue (ex. : faiblesse musculaire, somnolence) & 9 & $75 \%$ \\
Pertes d'équilibre (ex. : étourdissements, vertige, instabilité physique) & 7 & $58 \%$ \\
Troubles de la mémoire & 7 & $58 \%$ \\
Apathie (ex. : manque de motivation, d'initiative) & 7 & $58 \%$ \\
Trouble du langage & 4 & $33 \%$ \\
Confusion (ex. : désorientation, troubles de la conscience, sentiment « d'étrangeté ») & 4 & $33 \%$ \\
Incapacités physiques (ex. : maux de dos, amputation d'un membre) & 4 & $33 \%$ \\
Troubles visuels (ex. : vision double, perte de champ visuel) & 3 & $25 \%$ \\
Irritabilité & 3 & $25 \%$ \\
Sensibilité au bruit & 2 & $16 \%$ \\
Ralentissement (des mouvements) & 2 & $16 \%$ \\
Perte de l'odorat & 1 & $8 \%$ \\
\hline
\end{tabular}


Plusieurs de ces projets ont été identifiés dès le départ par le participant, alors que d'autres ont pris forme au fur et à mesure des activités réalisées et des interactions avec l'accompagnateur. Ces projets et activités initialement ciblés ont pu évoluer chez la plupart en fonction des nouvelles capacités et intérêts des participants. Ils se sont concrétisés dans diverses activités : marches, activités sportives avec l'accompagnateur, sorties extérieures, culturelles et récréatives (ex. visites de musées, sorties au restaurant, cinéma), déplacements à pied ou en transports collectifs pour se rendre à l'activité ciblée. Plusieurs activités de la vie quotidienne (ex. emplettes, rendez-vous médicaux) ou à visées relationnelles (échanges, confidences) ont aussi été réalisées, à domicile ou à l'extérieur du domicile (Tableau 3).

Les résultats montrent que plusieurs de ces activités ont eu des retombées positives. En effet, au terme de l'expérimentation, les effets principaux de l'APIC sur la participation sociale, concernent : (1) une meilleure assurance et un plus grand sentiment d'indépendance pour se réaliser (sphère des activités de la vie quotidienne); (2) un goût retrouvé d'être actif et de s'investir dans des activités de loisirs signifiantes (sphère des occupations et des loisirs); et (3) une plus grande ouverture envers de nouvelles possibilités de relations (sphère des relations avec les autres). Le Tableau 4 présente une synthèse de ces résultats.

Tableau 3. Sphère d'activités de participation sociale, exemples d'activités associées, nombre et pourcentage de participants mentionnant le type d'activités.

\begin{tabular}{|c|c|c|c|c|}
\hline $\begin{array}{l}\text { Sphères d'activités } \\
\text { de participation } \\
\text { sociale }\end{array}$ & $\begin{array}{l}\text { Types d'activités } \\
\text { réalisées avec } \\
\text { l'accompagnateur }\end{array}$ & Exemples & $N$ & $\%$ \\
\hline \multirow[t]{2}{*}{$\begin{array}{l}\text { Activités de la vie } \\
\text { quotidienne }\end{array}$} & Tâches domestiques & $\begin{array}{l}\text { Ramassage des feuilles dans la } \\
\text { cour, dégagement de la } \\
\text { neige, préparation d'un } \\
\text { repas, montage d'un bureau, } \\
\text { changement de pneus }\end{array}$ & 3 & $27 \%$ \\
\hline & $\begin{array}{l}\text { Activités liées à la } \\
\text { survie }\end{array}$ & $\begin{array}{l}\text { Emplettes, magasinage, } \\
\text { transactions bancaires, } \\
\text { visites médicales, prise } \\
\text { d'informations sur les } \\
\text { services }\end{array}$ & 8 & $73 \%$ \\
\hline \multirow[t]{2}{*}{$\begin{array}{l}\text { Occupations et } \\
\text { loisirs }\end{array}$} & $\begin{array}{l}\text { Activités récréatives/ } \\
\text { intellectuelles/ } \\
\text { culturelles à domicile }\end{array}$ & $\begin{array}{l}\text { Cartes, dominos, Scrabble, } \\
\text { utilisation de l'ordinateur, } \\
\text { lecture de la Bible }\end{array}$ & 8 & $73 \%$ \\
\hline & $\begin{array}{l}\text { Activités récréatives/ } \\
\text { intellectuelles/ } \\
\text { culturelles à } \\
\text { l'extérieur du } \\
\text { domicile }\end{array}$ & $\begin{array}{l}\text { Marcher, jouer un tennis, se } \\
\text { baigner, visites de musées, } \\
\text { cinéma, jardin botanique, } \\
\text { zoo, feux d'artifice, bingo }\end{array}$ & 10 & $91 \%$ \\
\hline \multirow[t]{2}{*}{$\begin{array}{l}\text { Relations avec les } \\
\text { autres }\end{array}$} & $\begin{array}{l}\text { Activités à visées } \\
\text { relationnelles } \\
\text { (échanges, soutiens } \\
\text { émotionnels) à } \\
\text { domicile }\end{array}$ & $\begin{array}{l}\text { Parler de ses inquiétudes, } \\
\text { conflits avec les proches, } \\
\text { décès, stress, événements du } \\
\text { passé, limitations associées } \\
\text { au TCC }\end{array}$ & 6 & $55 \%$ \\
\hline & $\begin{array}{l}\text { Activités à visées } \\
\text { relationnelles à } \\
\text { l'extérieur du } \\
\text { domicile }\end{array}$ & $\begin{array}{l}\text { Prendre un café, manger au } \\
\text { restaurant, fréquenter un café } \\
\text { pour socialiser }\end{array}$ & 5 & $46 \%$ \\
\hline
\end{tabular}


Tableau 4. Effets de l'APIC dans les trois sphères d'activités de la participation sociale.

\begin{tabular}{|c|c|}
\hline Sphères d'activités & Progression \\
\hline $\begin{array}{l}\text { Activités de la vie } \\
\text { quotidienne }\end{array}$ & $\begin{array}{l}\text { Plus grande assurance pour se réaliser : } \\
\text { Meilleure estime de soi et satisfaction personnelle } \\
\text { Plus grande confiance dans ses capacités à réaliser des tâches } \\
\text { Plus grande appétence pour la vie : } \\
\text { Attitude plus légère et confiante } \\
\text { Sentiment de mieux-être amélioré } \\
\text { Goût retrouvé à la vie, pour accomplir des tâches } \\
\text { Meilleur sentiment d'indépendance : } \\
\text { Prise d'initiatives personnelles }\end{array}$ \\
\hline Occupations et loisirs & $\begin{array}{l}\text { Plus grande assurance et motivation pour se mettre en mouvement : } \\
\text { Moins d'insécurité et d'inquiétudes lors de déplacements } \\
\text { Moins de crainte de chuter } \\
\text { Confiance dans ses capacités de réaliser seul une activité, à planifier et } \\
\text { réaliser des sorties extérieures } \\
\text { Goût retrouvé d'être actif physiquement } \\
\text { Reprise d'activités physiques } \\
\text { Récupération de capacités physiques : } \\
\text { Capacité de parcourir des distances longues et durée de l'activité plus } \\
\text { importante } \\
\text { Plus de stabilité physique } \\
\text { Moins d'essoufflements } \\
\text { Besoin moins fréquent de faire des arrêts pour se reposer }\end{array}$ \\
\hline $\begin{array}{l}\text { Relations avec les } \\
\text { autres }\end{array}$ & $\begin{array}{l}\text { Mieux-être personnel, réconfort soulagement } \\
\text { Ressentir un soulagement, un réconfort, se sentir moins seul, plus satisfait } \\
\text { du fait de pouvoir parler avec l'accompagnateur } \\
\text { Ouverture à de nouvelles possibilités de relations : } \\
\text { Renouer avec des proches et faire des activités avec eux ou avec des } \\
\text { connaissances } \\
\text { Plus grande facilité à communiquer avec les autres dans un contexte } \\
\text { social } \\
\text { Inclinaison à organiser des activités sociales ou à s'inscrire de nouveau } \\
\text { dans des contextes sociaux } \\
\text { Renouer une habitude de fréquentation d'un lieu de socialisation }\end{array}$ \\
\hline
\end{tabular}

Une meilleure assurance et un plus grand sentiment d'indépendance pour se réaliser, prendre des initiatives

L'apport de l'accompagnement-citoyen dans la sphère des activités de la vie quotidienne a été moins de redonner confiance aux participants dans leurs capacités à accomplir des tâches spécifiques - domestiques ou liées à la survie - que de les amener à développer une plus grande assurance dans leurs capacités à se réaliser. En effet, bien que de nombreuses séances d'accompagnement aient pris la forme d'une assistance ou d'une présence rassurante pour la réalisation de tâches domiciliaires (Tableau 3), les participants ont à peine souligné qu'ils avaient retrouvé une motivation à s'investir de nouveau dans ces tâches grâce au soutien d'accompagnement. Seuls quelques-uns ont noté qu'ils avaient retrouvé le goût pour la préparation de repas, tâche pour laquelle ils avaient perdu la motivation et d'autres, pour l'accomplissement, à l'occasion, de certaines tâches domiciliaires (ex. laver son balcon, coudre, épousseter).

De façon manifeste cependant, les participants ont développé un plus grand sentiment de confiance en soi et d'indépendance dans leur situation de vie. Plusieurs présentaient, 
au départ, des signes de replis sur soi, un manque d'appétence face à la vie, des sentiments d'insuffisance et une dépréciation de soi, le fait de moins sortir de chez soi, d'avoir moins de loisirs de même que l'ennui, ayant pour effet de démoraliser et de dévaloriser (Tableau 3). L'apathie ou le manque d'appétence à la vie était évoqué à répétition par plusieurs et se répercutait de façon extrême chez certains, qui éprouvaient des difficultés à sortir du lit ou avaient tendance à vouloir fréquemment dormir durant la journée. Peu à peu, des participants ont paru présenter une attitude plus légère ou se sont dits plus confiants dans leur situation de vie, recommençant à prendre des initiatives personnelles. Le fait d'avoir pu porter un regard réflexif sur leurs forces et leurs limites, d'avoir réalisé avec leur accompagnateur différentes AVQ (ex. préparer un de repas, faire le tri de vêtements, aider à se vêtir, réparer une lampe, réaliser ses emplettes, aller à ses rendez-vous médicaux), d'avoir été stimulés pour se mettre en action, a paru amener plusieurs à se reconstruire et à retrouver goût à la vie. Ces transformations ont eu un retentissement, tant dans la sphère des loisirs que dans celles des relations avec les autres, les acquis réalisés dans chacune des sphères d'activités de participation sociale s'interinfluençant (Tableau 5).

\section{Un goût retrouvé d'être actif et de s'investir dans des activités de loisirs signifiantes}

Grâce à l'accompagnement-citoyen, plusieurs participants ont retrouvé le goût d'être actifs, de se mettre en mouvement et de s'investir dans des activités signifiantes, ceci principalement à travers de nombreuses activités de loisirs extérieures. Ces activités ont en effet été très fréquentes au cours de l'expérimentation (Tableau 3) et ont rejoint le plus

Tableau 5. Sphère d'activités de la vie quotidienne : extraits de commentaires de participants.

\begin{tabular}{|c|c|}
\hline Pré-intervention & Impact longitudinal \\
\hline $\begin{array}{l}\text { J'ai perdu cette confiance en moi. Cette qualité } \\
\text { de me sentir capable de faire des choses. Une } \\
\text { capacité que j'ai cultivée toute ma vie et que } \\
\text { j'ai perdue. Alors, OK c'est perdu, mais peut- } \\
\text { on la récupérer? Ma mémoire décline. Ce } \\
\text { matin, j'avais mes gants, je travaillais et tout } \\
\text { à coup je n'avais qu'un gant. Je ne savais } \\
\text { plus où était l'autre. J'ai passé du temps à } \\
\text { chercher le gant et l'ai retrouvé dans un } \\
\text { endroit que je n'aurais jamais pensé. Ça c'est } \\
\text { très frustrant (12). }\end{array}$ & $\begin{array}{l}\text { C'est grâce à mon accompagnateur que je suis } \\
\text { ce que je suis aujourd'hui. Je suis } \\
\text { reconnaissant. J'ai un ange gardien qui m'a } \\
\text { gardé en vie. Mais ça prend du temps et on } \\
\text { finit par accepter certaines choses. Et ça je } \\
\text { l'ai accepté (12). }\end{array}$ \\
\hline $\begin{array}{l}\text { Je n'ai jamais eu autant de sensibilité comme je } \\
\text { l'ai maintenant. Ma vie a tout changé. Je ne } \\
\text { suis pas le même que j'étais avant. Le } \\
\text { courage, l'amour pour la vie, l'estime de moi, } \\
\text { la valorisation que je donnais à tout ce que je } \\
\text { faisais... Mon comportement a changé (14). }\end{array}$ & $\begin{array}{l}\text { C'est une présence importante parce qu'il y a } \\
\text { des choses que je peux faire seul, mais il y a } \\
\text { un danger. Par exemple, si je vais dégager les } \\
\text { feuilles qui se sont accumulées sur le toit, il y } \\
\text { a un risque. Alors je lui demande de tenir } \\
\text { l'échelle et je monte. Ça me donne beaucoup } \\
\text { plus de sécurité que d'être seul. Et, que } \\
\text { l'échelle, l'escalier puisse glisser. Et que je } \\
\text { me retrouve avec les jambes cassées (14). } \\
\text { Quand Lucie vient, j'ai beaucoup plus confiance } \\
\text { en moi. Parce que je parle. Parce que quand } \\
\text { on a quelque chose, il faut parler à } \\
\text { quelqu'un, ça débouche la tête (15). }\end{array}$ \\
\hline
\end{tabular}


grand nombre de participants $(N=9)$. Elles ont donné lieu à de nombreux déplacements, à pied ou en transports collectifs. Au total, près de 70 marches ont été effectuées avec les accompagnateurs, auxquelles s'ajoutent plusieurs déplacements à l'extérieur, soit pour se rendre dans un parc à proximité pour jouer aux dominos, pique-niquer, échanger, prendre un café ou comme moyen pour arriver à destination pour jouer au bingo, visionner un film, visiter un musée.

Peu à peu, plusieurs participants ont témoigné d'une plus grande assurance dans leurs déplacements et de meilleures capacités physiques. Certains ont remarqué qu'ils se déplaçaient plus facilement, qu'ils arrivaient à parcourir des distances plus longues en marchant, que la durée de l'activité était de plus en plus importante. D'autres se sentaient moins essoufflés, moins fatigués, avaient moins besoin de faire des arrêts fréquents en pratiquant des sports. Des participants se sont sentis plus assurés dans leurs déplacements (à l'aide d'une canne, d'une marchette), craignant moins de chuter.

Le fait de bouger, d'être en mouvement, de se déplacer à l'extérieur du domicile a manifestement eu des effets bénéfiques chez plusieurs. Peu à peu, des participants se sont sentis plus à l'aise pour pratiquer, seuls, une activité physique (ex. baignade) ou ont été plus confiants pour planifier et réaliser eux-mêmes des sorties extérieures (ex. organisation du transport adapté). D'autres se sont peu à peu déplacés vers l'activité de leur choix sans la présence de l'accompagnateur ou ont commencé à prendre de nouvelles initiatives de loisirs dans leur communauté. Dans le cours de son accompagnement, une femme âgée a recommencé à planifier une activité régulière de marche avec une amie. Une autre s'est inscrite à un cours de yoga. Un homme âgé a recommencé à sortir de son domicile et à socialiser dans la salle commune de sa résidence. Une femme âgée a repris ses exercices physiques dans un gymnase et une autre, s'est trouvé un travail à temps partiel (Tableau 6).

\section{Une plus grande ouverture vers de nouvelles possibilités de relations}

Tous les participants ont exprimé des sentiments de solitude et d'isolement importants, des besoins criants d'échanger avec leur accompagnateur, de s'épancher sur leurs difficultés. Le plus souvent sollicités par le participant lui-même, ces échanges permettaient de répondre à un besoin d'être entendu sur des inquiétudes, des émotions (ex. sentiment d'isolement, ennui, tristesse, événements douloureux du passé) ou des contentieux familiaux (ex. dépendance aux jeux du fils) ou encore, de s'exprimer sur des événements que certains évitaient d'aborder avec leurs proches.

Ces occasions échanges ont été déterminantes chez tous, le fait de pouvoir parler, apportant mieux-être, réconfort, soulagement et surtout, elles ont paru résonner dans la vie familiale et sociale. En outre, des participants ont recommencé à renouer des liens plus étroits avec des membres de leur famille immédiate avec qui ils avaient peu de contacts, à s'engager davantage dans des activités avec eux ou avec des connaissances (ex. sorties au restaurant, planification de voyages). D'autres se sont peu à peu inscrits dans un réseau de relations plus étendu ou ont paru retrouver le goût d'interagir dans des contextes sociaux. Dans le cours de son accompagnement, une femme âgée qui avait tendance à s'isoler au centre de jour qu'elle fréquente a commencé à communiquer plus facilement avec les autres. Un homme âgé s'est rendu disponible pour organiser des activités sociales dans sa résidence. Un autre a recommencé à fréquenter un café chilien dans sa communauté, ce qui lui a permis de se créer un nouveau réseau social. 
Tableau 6. Sphère des occupations et des loisirs : extraits de commentaires de participants.

\begin{tabular}{ll}
\hline Pré-intervention & Impact longitudinal \\
\hline Je fais des gros efforts pour retourner au & But even if Lucie is not there I go by myself and \\
gymnase, mais ce n'est pas... Je ne fais rien & I am careful and that's it. I can't stay home no \\
dans le fond. Je reste toujours dans ma & more no, no. Since that event happened, I \\
chambre, avec mon internet. Mais parce que & passed quite a few months at home and I said \\
là tu vois, ce qui me dérange c'est que je suis & no more, no more. Finished. That's it (15). \\
tannée de l'ordinateur : qu'est-ce que je vais & \\
faire? (3) & \\
Ca a toujours été assez bon. La participation & \\
côté responsabilité, c'était plus. Dans les & \\
classes, souvent j'étais dans les conseils de & \\
classe. Si on voulait aller montrer quelque & \\
chose au tableau dans les mathématiques ou & \\
l'algèbre, ça me faisait plaisir d'y aller. Pas & \\
dans tout. Je n'étais ni le boute-en-train ni & \\
l'organisatrice. & \\
J'étais très actif physiquement et tout d'un coup, \\
presque 2 ans, j'ai rien fait. Mes os, mes \\
muscles, tout est chambardé. Et ça a besoin \\
de reprendre graduellement. En plus, je jouais \\
au tennis 4 fois par semaine. De 2 à 3 heures, \\
4 heures à chaque fois. Dans la soirée, je \\
jouais au badminton. En hiver, je prenais une \\
gang de mon âge et j'allais faire du ski alpin. \\
Et tout s'est arrêté depuis mon TCC (14).
\end{tabular}

\section{Discussion}

Cet article avait pour but de présenter les impacts longitudinaux d'un accompagnement personnalisé à l'intégration communautaire (APIC) sur la participation sociale d'aînés ayant un traumatisme craniocérébral (TCC). L'étude a permis de montrer que, malgré un contexte initial limitatif, tous ont été en mesure d'identifier et de s'investir dans des activités qu'ils avaient à cœur. Également, tous ont progressé sur le plan de leur participation sociale en fonction de leurs propres capacités et intérêts dans l'une et l'autre des trois sphères d'activités de participation sociale du cadre de référence, les unes et les autres sphères s'interinfluençant. Le climat relationnel de confiance instauré avec l'accompagnateur, les possibilités offertes aux participants de s'exprimer et d'être reconnus dans leurs difficultés, de même que les nombreuses activités réalisées à domicile et surtout à l'extérieur du domicile, ont eu des répercussions d'ensemble et réciproques, créant une ouverture à l'autre, un goût retrouvé de se mettre en action et de se réaliser. De plus, des contributions spécifiques à chacune des sphères d'activités du cadre de référence ont pu être dégagées.

D'abord, les gains les plus significatifs se sont manifestés par des transformations internes sur le plan de l'identité personnelle, se traduisant peu à peu par une meilleure assurance et un plus grand sentiment d'indépendance dans sa situation de vie. La reconstruction de l'identité personnelle (Levack, Kayes, et Fadyl, 2010; Levasseur, Tribble, et Desrosiers, 2009) est reconnue d'ailleurs comme un projet de réadaptation fondamental chez la personne ayant un TCC. Cette reconstruction suppose un investissement important chez l'individu dans des activités exigeantes qui lui demandent de surmonter des défis nombreux, qui l'amènent à revoir la façon dont il se définit lui- 
même. Si le soutien des proches et des amis est alors indispensable, il appert que l'accompagnateur citoyen peut aussi tenir un rôle essentiel, en plaçant l'aîné au cœur du processus, en tablant sur ses potentialités, en sollicitant ses ressources et ses compétences (Vrancken et Bartholomé, 2004). Toutefois, peu de progrès ont été constatés au niveau de l'accomplissement de tâches domiciliaires, dont la préparation de repas - activité valorisée par les aînés (Provencher, Desrosiers, Demers, et Carmichael, 2015). Si le déclin dans la prise en charge des tâches domiciliaires est une réalité indiscutable tant chez la population générale âgée que chez les aînés atteints d'un TCC (Roy-Bouthot et al., 2014), on aurait pu, malgré tout, s'attendre à un investissement plus important de la part des participants dans la sphère domestique, surtout considérant le nombre relativement important d'activités domiciliaires réalisées avec les accompagnateurs. Le fait que la forte majorité des participants ait exprimé, dès le départ et de façon persistante tout au long de l'accompagnement, un besoin plus fondamental de bouger, d'être en mouvement, peut expliquer ces faibles progrès observés dans la réalisation des AVQ.

Une autre contribution de l'APIC a été d'amener les participants à bouger et à être actifs physiquement, à s'inscrire dans des activités de loisirs à l'extérieur du domicile, ce qui a eu des effets positifs sur le plan de leur participation sociale. Plusieurs ont pratiqué la marche avec leur accompagnateur, se sont déplacés avec lui pour se rendre à une activité de leur choix, ou ont réalisé avec lui de nombreuses activités de loisirs extérieures. Peu à peu, certains ont indiqué qu'ils avaient gagné en assurance dans leurs déplacements et au niveau de capacités physiques, remarquant qu'ils se déplaçaient plus facilement, craignant moins de chuter, se sentant moins facilement essoufflés et plus résistants. Les participants ont témoigné d'une diminution de pertes d'équilibre et de fatigue, séquelles du TCC fréquemment identifiés dans les écrits scientifiques chez des adultes atteints d'un TCC sans égard à l'âge comme atteintes limitant la participation sociale (Andersson et Finset, 1999; Brown, Gordon, et Spielman, 2003; Draper, Ponsford, et Schönberger, 2007; Dumont, 2003; Finset et Anderson, 2000; Katz, White, Alexander, et Klein, 2004; McFayden et al., 2009; McIntyre et al., 2014; SAAQ, 2003; Sendroy-Terrill et al., 2010; Yi et Dams-O'Connor, 2013). Ces gains ont permis à certains de s'autodéterminer davantage dans leur projet de participation sociale : les uns se sentant peu à peu plus à l'aise pour se déplacer seuls, pratiquer des sports ou des activités de détente sans la présence de l'accompagnateur; les autres, recommençant à planifier et réaliser eux-mêmes des activités extérieures. L'importance de se maintenir en mouvement n'est plus à démontrer chez l'aîné (Adams, Leibbrandt, et Moon, 2011; Hanson et al., 2013; Richard, Gauvin, Gosselin, et Laforest, 2008). Des niveaux plus élevés de participation sociale ont été observés chez des participants âgés qui pratiquaient fréquemment la marche (à chaque jour) et utilisaient souvent les transports collectifs (au moins une fois par semaine) (Richard et al., 2008). Le fait de pouvoir marcher dans un environnement physique et social sécuritaire en soutien aux déplacements a été identifié comme un facteur significatif, permettant à l'aîné de demeurer actif et engagé socialement, de participer à la vie dans sa communauté et de maintenir son indépendance (Hanson et al., 2013; Richard et al., 2008).

Enfin, une dernière contribution de l'APIC a été de créer de l'ouverture vers de nouvelles possibilités de relations sociales. Les participants ont d'abord été nombreux à solliciter des échanges auprès de leur accompagnateur et à reconnaître les bienfaits d'avoir pu s'exprimer avec lui sur des difficultés, inquiétudes, émotions (tristesse, colère), puis d'avoir pu s'épancher sur certains aspects plus sensibles de leur vie. Un 
lien de proximité relationnel étroit ainsi a paru s'établir entre accompagnateurs et aînés. Ce lien étroit a semblé d'autant plus bénéfique qu'un état d'exclusion de la vie sociale était observé chez tous les participants avant le début de l'intervention. Progressivement, des participants ont paru manifester une plus grande ouverture au plan relationnel, certains rétablissant des ponts avec des proches ou apparaissant plus ouverts à de nouvelles possibilités de relations sociales. Les uns ont semblé communiquer plus facilement dans des contextes sociaux, et les autres, ont recommencé à socialiser dans leur milieu de vie ou dans leur communauté (ex. organisation d'activités sociales dans sa résidence, fréquentation d'un café chilien). Les activités fondées sur les relations interpersonnelles, qu'elles se déroulent dans un cadre restreint entre l'accompagnateur et l'accompagné, avec des proches ou des connaissances ou dans la collectivité, sont considérées comme de puissants indicateurs de la participation sociale chez les aînés (Gilmour, 2012; Godelief, Mars, Gertrudis, et van Eijk, 2008; Levasseur et al., 2009; Sendroy-Terrill et al., 2010). C'est à travers des possibilités de relations sociales que l'aîné peut donner sens à sa vie, se créer des liens d'appartenance et exercer ses rôles sociaux (McLean et al., 2014; Raymond et al., 2008).

L'ensemble de ces résultats permet d'illustrer l'étendue des possibilités de participation sociale que peut soutenir l'accompagnateur-citoyen et surtout, amène à revisiter le concept dans le contexte d'aînés atteints d'un TCC. L'étude a montré que le fait d'échanger avec l'accompagnateur, de sortir du domicile pour faire des emplettes ou prendre un café dans un lieu public, constituaient autant d'activités signifiantes de participation sociale (Levasseur et al., 2010), offrant aux participants des possibilités de s'épanouir dans leur vie quotidienne, dans leurs loisirs et dans leurs interactions sociales (Hardaker, 2011; Levasseur et al., 2010; Raymond et al., 2008). Alors que dans sa définition classique, la participation sociale est appréhendée par l'engagement chez un individu dans une vie sociale organisée à l'extérieur de la famille, dans la communauté et dans les domaines de la vie civique et sociale (DahanOliel et al., 2008), le concept paraît mieux correspondre chez des aînés atteints d'un TCC à un engagement dans des activités multiples au domicile ou à l'extérieur du domicile, valorisées par eux, fondées sur des interactions avec les autres et réalisées en fonction des capacités et des intérêts (Levasseur et al., 2010). Ces résultats ouvrent la voie à de nouvelles avenues de recherche sur la manière dont l'accompagnement-citoyen peut contribuer à faire évoluer la participation sociale chez des aînés fragilisés, en cohérence avec leurs besoins fondamentaux (Brown et al., 2003; Gilmour, 2012).

\section{Forces et limites}

La rigueur scientifique de l'étude, étayée notamment par le haut niveau de saturation des données et la triangulation des analyses, a permis d'obtenir une compréhension approfondie des retombées de l'accompagnement APIC sur la participation sociale des participants âgés, ce qui en fait une étude originale et novatrice tant pour le secteur de la réadaptation que pour celui de la gériatrie et de la santé publique. La méthode d'échantillonnage utilisée appelle toutefois à une certaine prudence quant aux possibilités de généralisation des résultats produits. Il est plausible en effet que les participants de l'étude présentent un plus au niveau d'incapacités ou des caractéristiques personnelles qui les distinguent d'autres aînés atteints qui ont décliné l'invitation à participer à l'intervention d'accompagnement APIC (par manque de temps, parce qu'ils bénéficiaient d'un soutien satisfaisant, n'éprouvaient pas le besoin de recevoir un soutien à la participation sociale). Les données recueillies ne permettent pas d'apporter de précisions à ce sujet. 


\section{Conclusion}

Plusieurs interventions ont été déployées afin de favoriser la participation sociale des aînés, avec ou sans TCC, en soutenant la réalisation d'activités qui sont signifiantes pour eux. Au nombre des ressources humaines d'assistances présentement disponibles aux personnes atteintes d'un TCC, l'accompagnement APIC représente une ressource innovante centrée sur la promotion des forces de la personne, sur le développement de ses compétences et sur son autodétermination. Afin d'optimiser l'aide reçue, le programme APIC a été développé, implanté et évalué. Cette nouvelle modalité d'accompagnement contribue à l'amélioration de leur participation sociale. Les recherches futures permettront de mieux comprendre les caractéristiques du processus d'accompagnement ainsi que les particularités de ses effets comparativement à d'autres types d'intervention.

\section{Déclaration}

Aucun conflit d'intérêts potentiel n'a été rapporté par les auteurs.

\section{Funding}

This work was supported by the Fonds de recherche du Québec Santé

\section{Références}

Adams, K. B., Leibbrandt, S., et Moon, H. (2011). A critical review of the literature on social and leisure activity and wellbeing in later life. Ageing \& Society, 31(4), 683-712.

Andersson, S., et Finset, A. (1999). Apathy and depressed mood in acquired brain damage: Relationship to lesion localization and psychophysiological reactivity. Psychological Medicine, 29(2), 447-456.

Benker, D. (2003). Fear of falling in ambulatory older adults screening and interventions [Mémoire]. Washington, WA: Washington State University. Retrieved from https://research. libraries.wsu.edu/xmlui/handle/2376/3800

Brown, M., Gordon, W. A., et Spielman, L. (2003). Participation in social and recreational activity in the community by individuals with traumatic brain injury. Rehabilitation Psychology, 48(4), 266.

Buffel, T., Phillipson, C., et Scharf, T. (2012). Ageing in urban environments: Developing 'agefriendly' cities. Critical Social Policy, 32(4), 597-617.

Centers for Disease Control and Prevention (CDC). (2006). Web-based injury statistics query and reporting system. Retrieved from http://www.cdc.gov/traumaticbraininjury/index.html

Cisneros, E. (2012). Le traumatisme craniocérébral en âge avancé : particularités cliniques, réadaptation et accessibilité aux services. Psychologie Québec, 29(2), 44-47.

Dahan-Oliel, N., Gélinas, L., et Mazer, B. (2008). Social participation in the elderly: What does the literature tell us? Critical Review in Physical and Rehabilitation Medicine, 20(2), 159-176.

Draper, K., Ponsford, J.,et Schönberger, M. (2007). Psychosocial and emotional outcomes 10 years following traumatic brain injury. The Journal of Head Trauma Rehabilitation, 22(5), 278-287.

Dumont, C. (2003). L'identification des facteurs qui vont favoriser la participation sociale des adultes présentant des séquelles de traumatisme craniocérébral [Thèse]. Québec, Canada : Université Laval.

Dutil, É., Bier, N., et Gaudreault, C. (2006). Le Profil du Loisir, un instrument prometteur en ergothérapie. Canadian Journal of Occupational Therapy, 74(4), 326-336.

Dutil, É., Bottari, C., Vanier, M., et Rousseau, J. (2005). Profil des AVQ. Montréal : Les Éditions Emersion.

Faul, M., Wald, M. M., Xu, L., et Coronado, V. G. (2010). Traumatic brain injury in the United States; Emergency department visits, hospitalizations, and deaths, 2002-2006. Atlanta, GA: Centers for Disease Control and Prevention, National Center for Injury Prevention and Control. 
Finset, A., et Anderson, S. (2000). Coping strategies in patients with acquired brain injury: Relationships between coping, apathy, depression and lesion location. Brain Injury, 14(10), 887-905.

Frankel, J. E., Marwitz, J. H., Cifu, D. X., Kreutzer, J. S., Englander, J., et Rosenthal, M. (2006). A follow-up study of older adults with traumatic brain injury: Taking into account decreasing length of stay. Archives of Physical Medicine and Rehabilitation, 87(1), 57-62.

Gilmour, H. (2012). Social participation and the health and well-being of Canadian seniors. Health Reports, 23(4), 23-32.

Godelief, M. J., Mars, M. A., Gertrudis, I. J. M., et van Eijk, J. T. M. (2008). Characteristics of social participation as defined by older adults with a chronic physical illness. Disability and Rehabilitation, 30(17), 1298-1308.

Hammel, J., Magasi, S., Heinemann, A., Whiteneck, G., Bogner, J., et Rodriguez, E. (2008). What does participation mean? An insider perspective from people with disabilities. Disability and Rehabilitation, 30(19), 1445-1460.

Hanson, H. M., Schiller, C., Winters, M., Sims-Gould, J., Clarke, P., Curran, E., et Ashe, M. C. (2013). Concept mapping applied to the intersection between older adults' outdoor walking and the built and social environments. Preventive Medicine, 57(6), 785-791.

Hardaker, N. (2011). Pragmatic evidence based review. Community integration in moderate to severe traumatic brain injury. Wellington, New Zealand: Accident Compensation Corporation. Retrieved from https://www.acc.co.nz/assets/research/tbi-review-community.pdf

Katz, D. I., White, D. K., Alexander, M. P., et Klein, R. B. (2004). Recovery of ambulation after traumatic brain injury. Archives of Physical Medicine and Rehabilitation, 85(6), 865-869.

Lamontagne, M. E., Ouellet, M. C., et Simard, J. F. (2009). A descriptive portrait of human assistance required by individuals with brain injury. Brain Injury, 23(7-8), 693-701.

LeBlanc, J., de Guise, E., Gosselin, N., et Feyz, M. (2006). Comparison of functional outcome following acute care in young, middle-aged and elderly patients with traumatic brain injury. Brain Injury, 20(8), 779-790.

Lefebvre, H., et Levert, M. J. (2010). Pour une intervention centrée sur les besoins perçus de la personne et de ses proches. Dans I. Vincent, A. Loaëc, et C. Fournier (dirs.), Modèles et pratiques en éducation du patient : apports internationaux-5es Journées de la prévention (pp. 18-35). Paris : INPES.

Lefebvre, H., Levert, M. J., et Khelia, I. (2011). Un accompagnement personnalisé d'intégration communautaire en soutien au développement de la résilience : vers un modèle. Développement humain, handicap et changement social, 19(1), 103-110.

Lefebvre, H., Levert, M. J., Le Dorze, G., Croteau, C., Gélinas, I., Therriault, P. Y., et Samuelson, J. (2013). Un accompagnement citoyen personnalisé en soutien à l'intégration communautaire des personnes ayant subi un traumatisme craniocérébral : vers la résilience? Recherche en soins infirmiers, 4, 107-123.

Levack, W. M., Kayes, N. M., et Fadyl, J. K. (2010). Experience of recovery and outcome following traumatic brain injury: A metasynthesis of qualitative research. Disability and Rehabilitation, 32(12), 986-999.

Levasseur, M., Lefebvre, H., Levert, M. J., Lacasse-Bédard, J., Desrosiers, J., Therriault, P. Y., et Carbonneau, H. (2016). Personalized citizen assistance for social participation (APIC): A promising intervention for increasing mobility, accomplishment of social activities and frequency of leisure activities in older adults having disabilities. Archives of Gerontology and Geriatrics, 64, 96-102.

Levasseur, M., Richard, L., Gauvin, L., et Raymond, É. (2010). Inventory and analysis of definitions of social participation found in the aging literature: Proposed taxonomy of social activities. Social Science \& Medicine, 71(12), 2141-2149.

Levasseur, M., Tribble, D. S. C., et Desrosiers, J. (2009). Meaning of quality of life for older adults: Importance of human functioning components. Archives of Gerontology and Geriatrics, 49(2), e91-e100.

Levert, M. J., Gélinas, I., et Lefebvre, H. (2016). Pour un modèle régional d'offre de service en accompagnement [Rapport de recherche]. Montréal : Groupe interdisciplinaire de recherche sur la résilience et la réadaptation communautaire (GIRR).

Levert, M. J., Lefebvre, H., Levasseur, M., Gélinas, I., McKerral, M., Roy, O., et Proulx, M. (2017). Towards a better understanding of the impact of the sequelae of TBI on the social participation of seniors. Archives of Nursing Practice and Care, 3(3), 68-76.

McCluskey, A. (2000). Paid attendant carers hold important and unexpected roles which contribute to the lives of people with brain injury. Brain Injury, 14(11), 943-957. 
McColl, M. A., Carlson, P., Johnston, J., Minnes, P., Shue, K., Davies, D., et Karlovits, T. (1998). The definition of community integration: Perspectives of people with brain injuries. Brain Injury, 12(1), 15-30.

McFadyen, B. J., Cantin, J-F., Swaine, B., Duchesneau, G., Doyon, J., Dumas, D, et Fait, P. (2009). Modality-specific, multitask locomotor deficits persist despite good recovery after a traumatic brain injury. Archives of Physical Medicine and Rehabilitation, 90(9), 1596-1606. doi:10.1016/j.apmr.2009.03.010

McIntyre, A., Janzen, S., et Teasell, R. (2014). Traumatic brain injury in older adults: A review. Topics in Geriatric Rehabilitation, 30(3), 230-236.

McIntyre, A., Mehta, S., Janzen, S., Aubut, J., et Teasell, R. W. (2013). A meta-analysis of functional outcome among older adults with traumatic brain injury. NeuroRehabilitation, 32(2), 409-414.

McLean, A. M., Jarus, T., Hubley, A. M., et Jongbloed, L. (2014). Associations between social participation and subjective quality of life for adults with moderate to severe traumatic brain injury. Disability and Rehabilitation, 36(17), 1409-1418.

Mucchielli, A. (2005). Le développement des méthodes qualitatives et l'approche constructiviste des phénomènes humains. Recherches qualitatives, 1, 7-40.

Organisation mondiale de la santé. (2007). Guide mondial des violes-amies des ânés. Genève : Auteur.

Paillé, P., et Mucchielli, A. (2016). L'analyse qualitative en sciences humaines et sociales (4e éd). Paris: Armand Colin.

Perroux, M., Lefebvre, H., Levert, M. J., et Malo, D. (2013). Besoins perçus et participation sociale des personnes ayant un traumatisme crânien léger. Santé Publique, 25(6), 719-728.

Pourtois, J. P., et Desmet, H. (2014). L'éducation implicite. Paris : Presses universitaires de France.

Provencher, V., Desrosiers, J., Demers, L., et Carmichael, P. H. (2016). Optimizing social participation in community-dwelling older adults through the use of behavioral coping strategies. Disability and Rehabilitation, 38(10), 972-978.

Raymond, É., Gagné, D., Sévigny, A., et Tourigny, A. (2008). La participation sociale des aînés dans une perspective de vieillissement en santé. Réflexion critique appuyée sur une analyse documentaire. Montréal : Institut national de santé publique du Québec.

Richard, L., Gauvin, L., Gosselin, C., et Laforest, S. (2008). Staying connected: Neighbourhood correlates of social participation among older adults living in an urban environment in Montreal, Quebec. Health Promotion International, 24(1), 46-57.

Roy-Bouthot, K., Filliatrault, P., Caron, C., Gagnon, M., Prémont, S., et Levasseur, M. (2014). Modification of the assessment of life habits (LIFE-H m) to consider personalized satisfaction with participation in activities and roles: Results from a construct validity study with older adults. Disability and Rehabilitation, 36(9), 737-743.

Sendroy-Terrill, M., Whiteneck, G. G., et Brooks, C. A. (2010). Aging with traumatic brain injury: Cross-sectional follow-up of people receiving inpatient rehabilitation over more than $3 \mathrm{dec}-$ ades. Archives of Physical Medicine and Rehabilitation, 91(3), 489-497.

Société d'assurance-automobile du Québec (SAAQ). (2003). Le traumatisme craniocérébral. Brochure à l'intention des familles et des personnes atteintes. Québec : Gouvernement du Québec.

Stake, R. E. (2005). Qualitative case studies. In N. K. Denzin et Y. S. Lincoln (Eds.), The handbook of qualitative research (3rd ed., pp. 119-149). Thousand Oaks, CA: Sage Publications.

Table de concertation en accompagnement de Montréal. (2012). L'accompagnement : au-delà du domicile pour une réelle participation sociale [Rapport]. Montréal, Québec : Auteur.

Therriault, P. Y., Lefebvre, H., Guindon, A., Levert, M. J., et Briand, C. (2013). Accompagnement citoyen personnalisé en intégration communautaire : un défi pour la santé mentale? Santé mentale au Québec, 38(1), 165-188.

Vrancken, D., et Bartholomé, C. (2004). L'accompagnement des personnes handicapées en Belgique : un concept au coeur des nouvelles politiques sociales. Nouvelles pratiques sociales, 17(1), 98-111.

Winkler, D., Unsworth, C., et Sloan, S. (2006). Factors that lead to successful community integration following severe traumatic brain injury. The Journal of Head Trauma Rehabilitation, 21(1), 8-21.

Yi, A., et Dams-O'Connor, K. (2013). Psychosocial functioning in older adults with traumatic brain injury. NeuroRehabilitation, 32(2), 267-273. 